\title{
Various breathers and rogue waves for the coupled long-wave-short-wave system
}

\author{
Chuanjian Wang $^{1 *}$ and Zhengde Dai ${ }^{2}$
}

\author{
*Correspondence: \\ wcj20082002@aliyun.com \\ 'Department of Mathematics, \\ Kunming University of Science and \\ Technology, Kunming, Yunnan \\ 650500 , P.R. China \\ Full list of author information is \\ available at the end of the article
}

\begin{abstract}
Explicit forms of various breathers, including inclined periodic breather, Akhmediev breather, Ma breather and rogue wave solutions, are obtained for the coupled long-wave-short-wave system by using a Hirota two-soliton method with complex frequency and complex wave number. Based on the structures of these breather solutions and figures via computer simulation, the characteristics of various breather solutions are discussed which might provide us with useful information on the dynamics of the relevant physical fields.
\end{abstract}

Keywords: coupled long-wave-short-wave system; Hirota two-soliton method; breather; rogue wave

\section{Introduction}

It is well known that solitary wave solutions of nonlinear evolution equations play an important role in nonlinear science fields, especially in nonlinear physical science, since they can provide much physical information and more insight into the physical aspects of the problem and thus lead to further applications [1]. In recent years, rogue waves, as a special type of solitary waves, have triggered much interest in various physical branches. Rogue waves, alternatively called freak or giant waves, were first observed under circumstances of arbitrary depths of the ocean. One always has two or even more times higher amplitude than their surrounding waves and generally they form in a short time for which reason people think that it comes from nowhere [2,3]. Rogue waves have been the subject of intensive research in oceanography [2], superfluid helium [3], Bose-Einstein condensates [4], optical fibers [5], plasma physics [6], financial markets and related fields [7-9]. The first-order rational solution of the self-focusing nonlinear Schödinger equation (NLS) was first found by Peregrine to describe the rogue waves phenomenon [10]. Recently, by using the Darboux dressing technique or the Hirota bilinear method, rogue waves solutions in complex systems such as described by the Hirota equation, Sasa-Satsuma equation, Davey-Stewartson equation, coupled Gross-Pitaevskii equation, coupled NLS MaxwellBloch equation and so on have been demonstrated [11-18].

Now we consider the following coupled long-wave-short-wave system [19-22]:

$$
\left\{\begin{array}{l}
i p_{t}-2 p_{x x}+2 p(b-\omega)=0, \\
i q_{t}-2 q_{x x}+2 q(b-\omega)=0, \\
b_{t}-4\left(p q^{*}+p^{*} q\right)_{x}=0 .
\end{array}\right.
$$

O2014 Wang and Dai; licensee Springer. This is an Open Access article distributed under the terms of the Creative Commons Attribution License (http://creativecommons.org/licenses/by/2.0), which permits unrestricted use, distribution, and reproduction in any medium, provided the original work is properly cited. 
In the above equation, $p(x, t)$ and $q(x, t)$ are the orthogonal components of the envelope of a rapidly varying complex field (the short-wave) representing a transverse wave whose group velocity resonates with the phase velocity of a real field $b(x, t)$ (the long wave) representing a longitudinal wave. The $*$ denotes complex conjugation and $\omega$ is an arbitrary real constant. The CLS equations (1.1) generalize the scalar long-wave-short-wave resonance equations derived by Djordjevic and Redekopp [19] for long-wave-short-wave interactions when the more generic nonlinear Schrödinger equation breaks down due to a singularity in the coefficient of the cubic nonlinearity; the dispersion of the short-wave is balanced by the nonlinear interaction of the long wave, while the self-interaction of the short-wave drives the evolution of the long wave. Other studies of long-wave-short-wave interactions include those by Benney [20] and Grimshaw [21]. The CLS equations (1.1) are integrable in the sense that they possess an equivalent scattering problem formulation as a Lax pair of commuting differential operators on a subalgebra of $s l(4)$. Wright III [22] has obtained an auto-Bäklund transformation for plane-wave solutions of a system of coupled long-waveshort-wave equations by using the dressing method. The spatially periodic orbits on a homoclinic manifold of a torus of spatially independent plane waves were constructed by evaluating the auto-Bäklund transformation.

\section{Hirota two-soliton method and various breathers}

In this section, we will use the Hirota two-soliton method [23, 24] to construct our result.

\subsection{Hirota two-soliton method}

Hirota two-soliton method was first proposed by Hirota [1]. For a general nonlinear partial differential equation in the form $P\left(u, u_{t}, u_{x}, \ldots\right)=0, P$ is a polynomial in its arguments. By Painlevé analysis, a transformation $u=T(f)$ is made for some new and unknown function $f$. By using the above transformation, the original equation can be converted into Hirota's bilinear form,

$$
G\left(D_{t}, D_{x} ; f\right)=0
$$

where the $D$-operator is defined by $[1]$

$$
D_{t}^{n} D_{x}^{m} f(x, y) \cdot g(x, t)=\left.\left(\frac{\partial}{\partial t}-\frac{\partial}{\partial t^{\prime}}\right)^{n}\left(\frac{\partial}{\partial x}-\frac{\partial}{\partial x^{\prime}}\right)^{m}\left[f(x, t) g\left(x^{\prime}, t^{\prime}\right)\right]\right|_{x^{\prime}=x, t^{\prime}=t} \text {. }
$$

So the solutions of original partial differential equation can be converted into the solutions of bilinear differential equations. We solve the above bilinear differential equations to get breather wave solutions by using a two-soliton method with the help of MAPLE.

Now we make the dependent variable transformation $[23,24]$ for the system (1.1):

$$
p=\frac{G}{F}, \quad q=\frac{H}{F}, \quad b=b_{0}-2(\ln F)_{x x},
$$

where $G, H$ are complex valued functions, $F$ is a real valued function, and $b_{0}$ is a constant. Then the system (1.1) can be rewritten as the following coupled bilinear differential 
equations for $F, G$, and $H$ :

$$
\left\{\begin{array}{l}
\left(i D_{t}-2 D_{x}^{2}+A\right) G \cdot F=0 \\
\left(i D_{t}-2 D_{x}^{2}+A\right) H \cdot F=0 \\
\left(D_{t} D_{x}-C\right) F \cdot F+4\left(G \cdot H^{*}+G^{*} \cdot H\right)=0
\end{array}\right.
$$

where $A=2\left(b_{0}-\omega\right)$ and $C$ is the integration constant. So the system (1.1) has been bilinearized. Then the solutions of the system (1.1) can be converted into the solutions of coupled bilinear differential equations (2.2).

The two-soliton solutions of bilinear differential equations can be expressed in the form

$$
\left\{\begin{array}{l}
G(x, t)=p_{0} e^{-I a t}\left(1+\delta_{1} e^{\eta_{1}}+\delta_{2} e^{\eta_{2}}+M \delta_{1} \delta_{2} e^{\eta_{1}+\eta_{2}}\right), \\
H(x, t)=q_{0} e^{-I a t}\left(1+\delta_{3} e^{\eta_{1}}+\delta_{4} e^{\eta_{2}}+M \delta_{3} \delta_{4} e^{\eta_{1}+\eta_{2}}\right), \\
F(x, t)=1+e^{\eta_{1}}+e^{\eta_{2}}+M e^{\eta_{1}+\eta_{2}}
\end{array}\right.
$$

where $\eta_{1}=a_{1} x+c_{1} t+r, \eta_{2}=a_{2} x+c_{2} t+r, \delta_{i}(i=1,2,3,4)$ are complex numbers and $a$, $p_{0}, q_{0}, \delta_{5}, \delta_{6}$ are real numbers. By comparing with the two-wave functions of the multiple exp-function algorithm [25], one concludes that they are the same.

In order to obtain the required breather solutions, we consider the case that $a_{i}, c_{i}(i=1,2)$ are complex numbers, that is, taking wave numbers and frequencies that are complex, respectively. Indeed, let $a_{1}=k_{2}-i k_{1}, a_{2}=k_{2}+i k_{1}, c_{1}=l_{2}-i l_{1}, c_{2}=l_{2}+i l_{1}$, substituting (2.3) into (2.2), we can obtain various breathers by restricting the parameters suitably. They can be rewritten in terms of trigonometric and hyperbolic functions. In the following, we report the explicit forms of these breather solutions.

\subsection{Various breathers of the coupled long-wave-short-wave system}

\subsubsection{Inclined periodic breather}

The inclined periodic breather expressions for $p, q, b$ can be expressed in the following form:

$$
\left\{\begin{array}{l}
p=p_{0} e^{-i(a t+\theta) \frac{\sqrt{M} \cosh \left(\zeta+\phi_{1}+r+i \theta\right)+\cos \left(\xi-i \phi_{2}\right)}{\sqrt{M} \cosh \left(\zeta+\phi_{1}+r\right)+\cos \xi}} \\
q=q_{0} e^{-i(a t+\theta) \frac{\sqrt{M} \cosh \left(\zeta+\phi_{1}+r+i \theta\right)+\cos \left(\xi-i \phi_{2}\right)}{\sqrt{M} \cosh \left(\zeta+\phi_{1}+r\right)+\cos \xi}} \\
b=b_{0}-\frac{2\left(d_{1} \cosh \left(\zeta+\phi_{1}+r\right) \cos \xi+d_{2} \sinh \left(\zeta+\phi_{1}+r\right) \sin \xi+d_{3}\right)}{\left(\sqrt{M} \cosh \left(\zeta+\phi_{1}+r\right)+\cos \xi\right)^{2}}
\end{array}\right.
$$

under the conditions

$$
\begin{aligned}
& \delta_{1}=\frac{l_{1}+2 k_{2}^{2}-2 k_{1}^{2}-i\left(4 k_{2} k_{1}-l_{2}\right)}{l_{1}+2 k_{1}^{2}-2 k_{2}^{2}+i\left(4 k_{2} k_{1}+l_{2}\right)}, \quad A=-a, \\
& \delta_{2}=\frac{l_{1}+2 k_{1}^{2}-2 k_{2}^{2}-i\left(4 k_{2} k_{1}+l_{2}\right)}{l_{1}+2 k_{2}^{2}-2 k_{1}^{2}+i\left(4 k_{2} k_{1}-l_{2}\right)}, \quad C=8 p_{0} q_{0} \text {, } \\
& M=1+\frac{4\left(k_{1}^{2}+k_{2}^{2}\right)^{3}}{\left(k_{2} l_{1}-l_{2} k_{1}\right)^{2}-4 k_{2}^{2}\left(k_{1}^{2}+k_{2}^{2}\right)^{2}}, \\
& 64 k_{1}\left(k_{1}^{2}-3 k_{2}^{2}\right) q_{0} p_{0} \\
& =8 k_{1} k_{2}\left(2 l_{2}\left(k_{1}^{2}-k_{2}^{2}\right)+3 k_{2} k_{1} l_{1}\right)-l_{1}\left(4\left(k_{1}^{4}+k_{2}^{4}\right)+3 l_{2}^{2}-l_{1}^{2}\right), \\
& 4 k_{1}^{7} l_{2}+28 k_{2} k_{1}^{6} l_{1}+l_{2}^{3} k_{1}^{3}+\left(84 k_{2}^{4}+9 l_{2}^{2}\right) l_{1} k_{2} k_{1}^{2}+9 l_{1}^{2} l_{2} k_{1} k_{2}^{2}+k_{2}^{3} l_{1}^{3}
\end{aligned}
$$




$$
\begin{aligned}
= & 36 k_{1}^{5} k_{2}^{2} l_{2}+140 k_{2}^{3} k_{1}^{4} l_{1}+\left(4 k_{2}^{4}+3 l_{1}^{2}\right) l_{2} k_{1}^{3}+3 k_{2} k_{1}^{2} l_{1}^{3} \\
& +\left(28 k_{2}^{4}+3 l_{2}^{2}\right) l_{2} k_{1} k_{2}^{2}+\left(3 l_{2}^{2}+4 k_{2}^{4}\right) l_{1} k_{2}^{3}, \\
\delta_{3}= & \delta_{1}, \quad \delta_{4}=\delta_{2},
\end{aligned}
$$

where $\zeta=k_{2} x+l_{2} t, \eta=k_{1} x+l_{1} t, d_{1}=\sqrt{M}\left(k_{2}^{2}-k_{1}^{2}\right), d_{2}=2 \sqrt{M} k_{1} k_{2}, d_{3}=M k_{2}^{2}-k_{1}^{2}, \phi_{1}=$ $\ln \sqrt{M}, \phi_{2}=\ln \left|\delta_{2}\right|, \theta=\arctan \frac{4\left(2 k_{2} k_{1} l_{1}-l_{2} k_{1}^{2}+l_{2} k_{2}^{2}\right)}{4\left(k_{1}^{2}+k_{2}^{2}\right)^{2}-l_{1}^{2}-l_{2}^{2}}$.

Obviously, the parameters $\delta_{1}, \delta_{2}$ have the following relation:

$$
\delta_{1} \delta_{2}^{*}=1
$$

Some asymptotic behaviors of the obtained solutions can be found. Without loss of generality, we assume that $l_{2}>0$ or $k_{2}>0$, and we obtain from (2.4)

$$
(p, q, b) \rightarrow\left(p_{0} e^{-i(a t-2 \theta)}, q_{0} e^{-i(a t-2 \theta)}, b_{0}\right), \quad \text { as } t \text { or } x \rightarrow+\infty,
$$

and

$$
(p, q, b) \rightarrow\left(p_{0} e^{-i a t}, q_{0} e^{-i a t}, b_{0}\right), \quad \text { as } t \text { or } x \rightarrow-\infty .
$$

Obviously, the limit solution $\left(p_{0} e^{-i(a t-2 \theta)}, q_{0} e^{-i(a t-2 \theta)}, b_{0}\right)$ is an exact plane-wave solution of (1.1). This means that in some limit, $t \rightarrow \pm \infty$ or $x \rightarrow \pm \infty$ or in both, this new solution will approach the original plane-wave solution, up to some phase shift. It is shown that this solution, given by (2.4), represents a kind of homoclinic solution and meanwhile contains a periodic wave $\cos \xi$ whose amplitude periodically oscillates with the evolution of time. So this solution represented by $(p, q, b)$ of $(2.4)$ is a homoclinic breather solution. The trajectory of these solutions is defined explicitly by

$$
x=-\frac{l_{2} t+\phi_{1}+r}{k_{2}},
$$

which can be derived from $\zeta+\phi_{1}+r=0$. So the solution in (2.4) evolves periodically along the straight line with a certain angle of $x$ axis and $t$ axis. Figure 1 illustrates the behavior of this inclined periodic breather solution, which is periodic both in space and in time. Figure 1 shows that the functions $p(x, t)$ and $q(x, t)$ have periodic bright solitary wave features, while $b(x, t)$ shows a periodic dark solitary wave feature. The periodic bright solitary waves have one upper peak and two caves in each periodic unit. However, the periodic dark solitary waves have two upper lumps and one deep cave in each periodic unit. What is more, in Figure 1 we can see that an obvious common feature of this solution $(p, q, b)$ is that it is a singular breather and describes a single wave in localized space and time in each periodic unit. Moreover, it is shown that those localized humps with peak amplitude of more than three times the non-zero constant background arise from the non-zero constant background and then disappear into the non-zero constant background again in the intermediate times. So this solution has a similar structure as the rogue waves in each periodic unit. Hence this solution is called a periodic rogue wave solution. If we let $a=0$, then $(p, q, b) \rightarrow\left(p_{0} e^{-2 \theta i}, q_{0} e^{-2 \theta i}, b_{0}\right)$, as $t \rightarrow+\infty$, and $(p, q, b) \rightarrow\left(p_{0}, q_{0}, b_{0}\right)$, as 


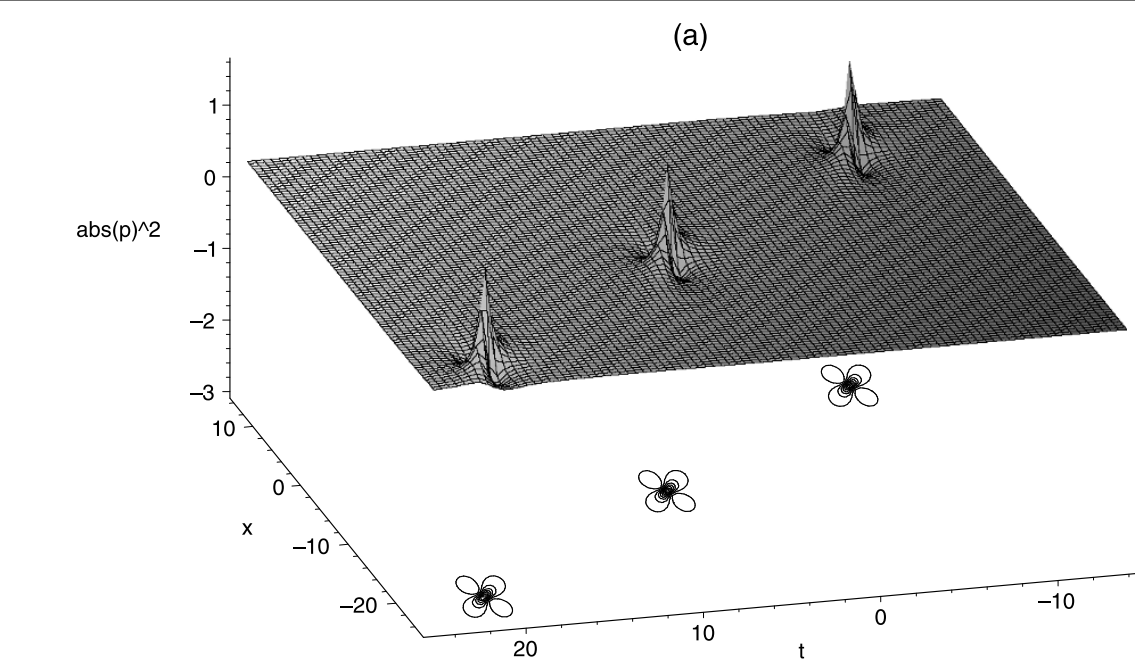

(b)

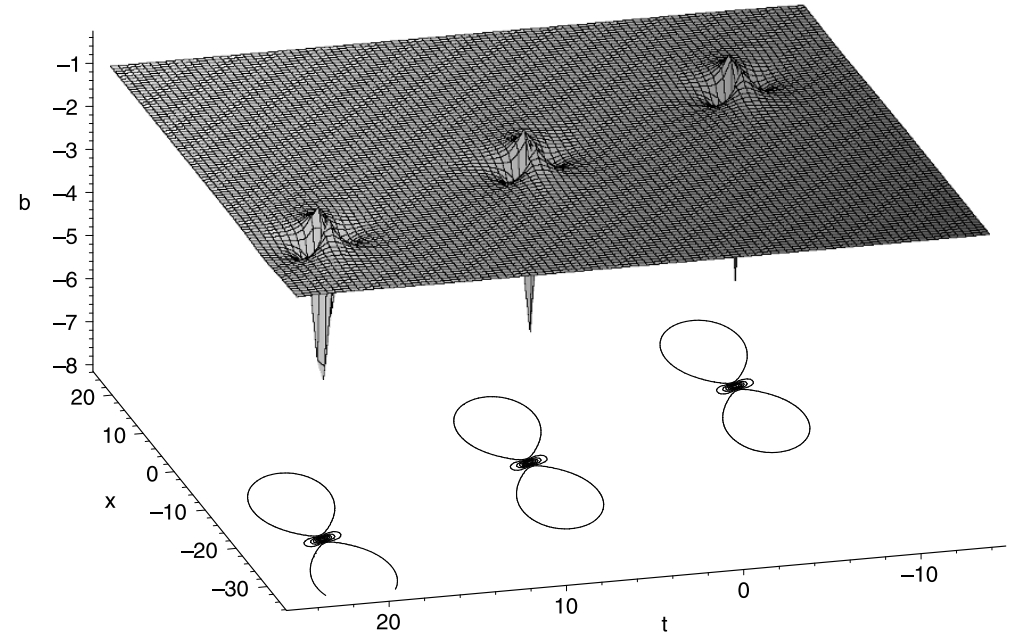

Figure 1 Inclined periodic breather profiles of $|\boldsymbol{p}(\boldsymbol{x}, \boldsymbol{t})|^{\mathbf{2}}(\mathbf{a})$ and $\boldsymbol{b}(\boldsymbol{x}, \boldsymbol{t})(\mathbf{b})$. The parameters are selected as $k_{2}=l_{2}=0.2, p_{0}=0.5, q_{0}=0.4, a=1, b_{0}=-1$. A similar profile occurs for $a$ also (not shown here). Curved lines drawn at the bottom of this figure are contour lines.

$t \rightarrow-\infty$, which is different. In this case, this solution is a heteroclinic breather solution. In fact, this solution is called a complexiton solution in Ref. [26].

From the inclined periodic breather solution we can derive the Akhmediev breather [27] (space periodic breather solution), Ma breather [28] (time periodic breather solution) and rogue wave solutions.

\subsubsection{Ma breather}

To obtain the Ma breather solution, we consider the choice $l_{2}=0$ in (2.4). In this case, $\zeta=0$ implies that the trajectory equation of these solutions can be expressed as $x=0$. So we can get another form of the breather solution which will propagate periodically along 
(a)

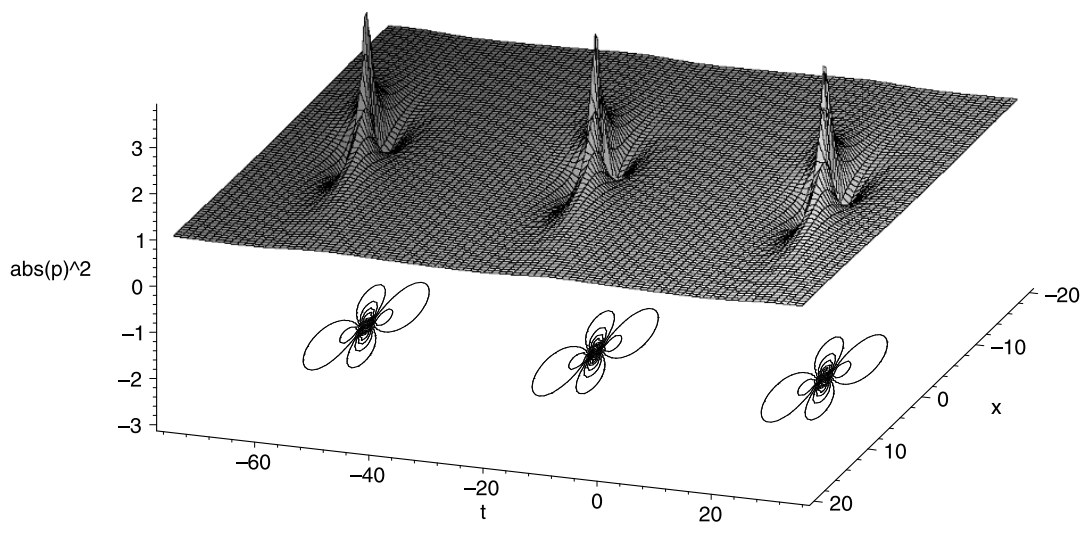

(b)

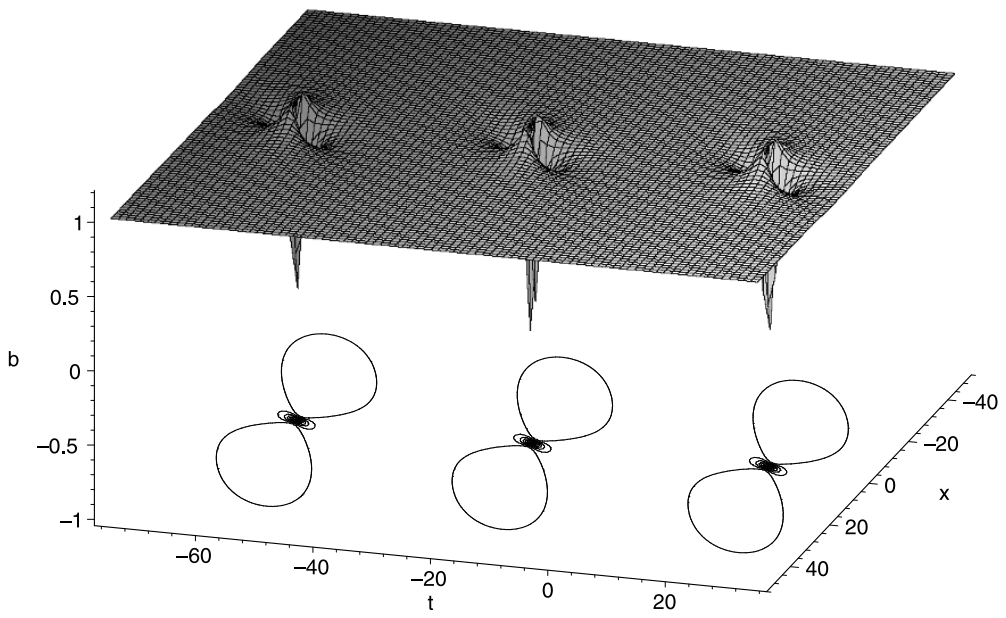

Figure 2 Ma breather profiles of $|\boldsymbol{p}(\boldsymbol{x}, \boldsymbol{t})|^{\mathbf{2}}$ (a) and $\boldsymbol{b}(\boldsymbol{x}, \boldsymbol{t})(\mathbf{b})$. The parameters are selected as $k_{2}=0.1$, $p_{0}=a=b_{0}=1, q_{0}=0.04$. A similar profile occurs for $|q|^{2}$ also (not shown here). Curved lines drawn at the bottom of this figure are contour lines.

the straight line with the $x$-axis, that is,

$$
\left\{\begin{array}{l}
p=p_{0} e^{-i(a t+\theta)} \frac{\sqrt{M} \cosh \left(k_{2} x+\phi+i \theta\right)+\cos \left(\left(k_{1} x+l_{1} t\right)-i \phi_{2}\right)}{\sqrt{M} \cosh \left(k_{2} x+\phi\right)+\cos \left(k_{1} x+l_{1} t\right)} \\
q=q_{0} e^{-i(a t+\theta)} \frac{\sqrt{M} \cosh \left(k_{2} x+\phi+i \theta\right)+\cos \left(\left(k_{1} x+l_{1} t\right)-i \phi_{2}\right)}{\sqrt{M} \cosh \left(k_{2} x+\phi\right)+\cos \left(k_{1} x+l_{1} t\right)} \\
b=b_{0}-\frac{2\left(d_{1} \cosh \left(k_{2} x+\phi\right) \cos \left(k_{1} x+l_{1} t\right)+d_{2} \sinh \left(k_{2} x+\phi\right) \sin \left(k_{1} x+l_{1} t\right)+d_{3}\right)}{\left(\sqrt{M} \cosh \left(k_{2} x+\phi\right)+\cos \left(k_{1} x+l_{1} t\right)\right)^{2}}
\end{array}\right.
$$

where $\phi=\phi_{1}+r$. This solution tends to the plane-wave solution as $x \rightarrow \infty$. We depict this solution (2.6) in Figure 2. The plot shows that this solution is periodic with period $\frac{2 \pi}{l_{1}}$ in $t$ and localized, exponentially decaying in the $x$ direction. This solution which is temporally breathing and spatially oscillating is called a Ma breather.

\subsubsection{Akhmediev breather}

To obtain the Akhmediev breather solution, we consider the choice $k_{2}=0$ in (2.4). In this case, $\zeta=0$ implies that the trajectory equation of these solutions can be expressed as $t=0$. So we can obtain another form of the breather solution which will propagate only in the 


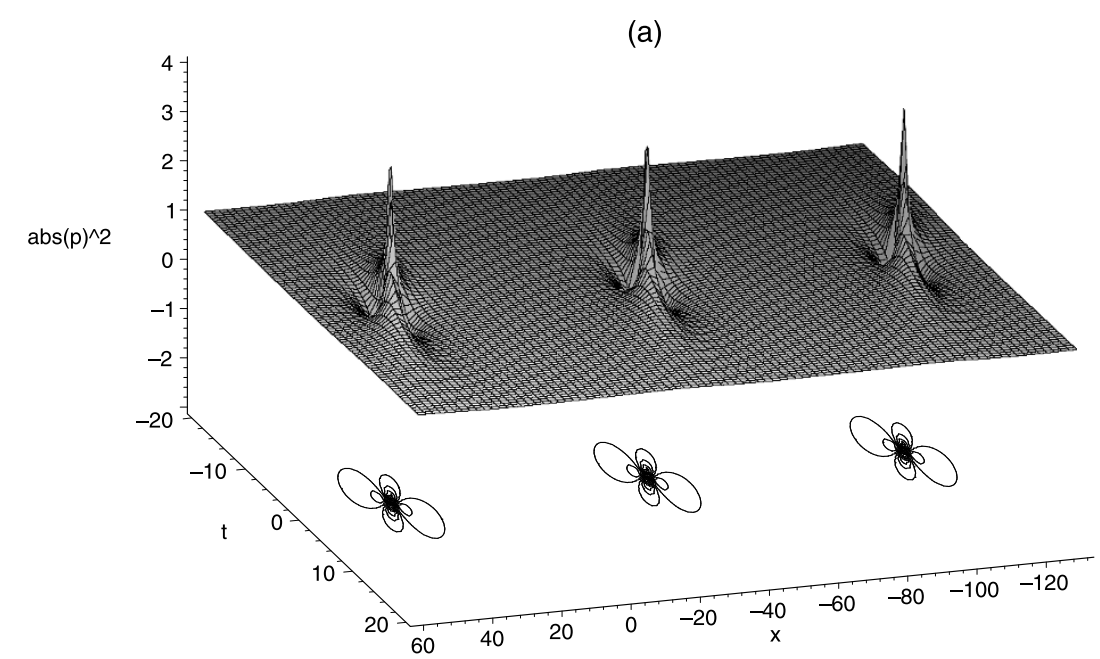

(b)

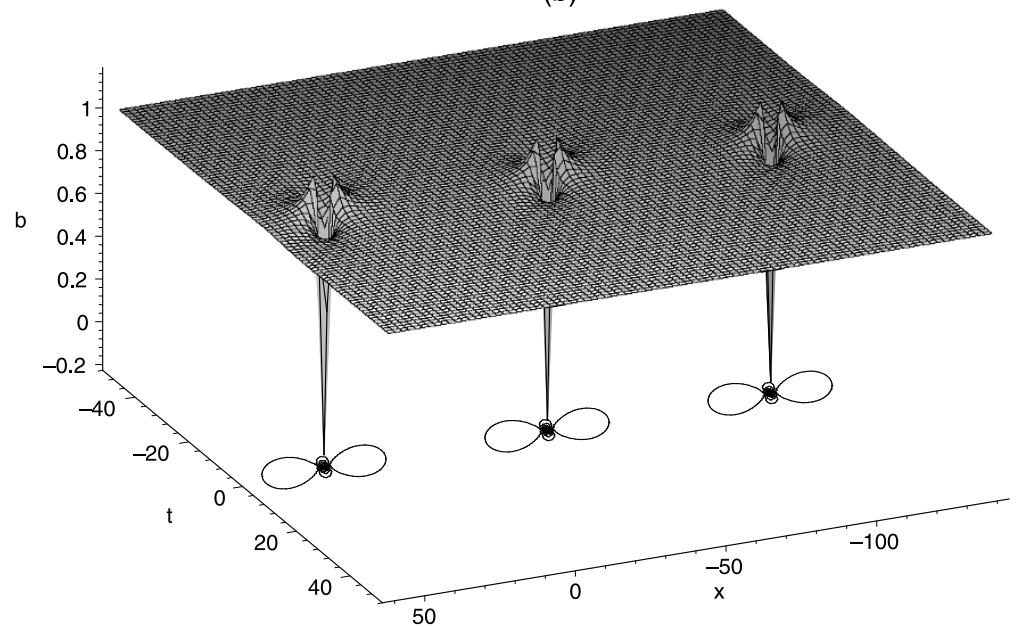

Figure 3 Akhmediev breather profiles of $|p(x, t)|^{2}(\mathbf{a})$ and $\boldsymbol{b}(\boldsymbol{x}, \boldsymbol{t})(\mathbf{b})$. The parameters are selected as $I_{2}=0.1, p_{0}=a=b_{0}=1, q_{0}=0.04$. A similar profile occurs for $|q|^{2}$ also (not shown here). Curved lines drawn at the bottom of this figure are contour lines.

time direction, that is,

$$
\left\{\begin{array}{l}
p=p_{0} e^{-i(a t+\theta)} \frac{\sqrt{M} \cosh \left(l_{2} t+\phi+i \theta\right)+\cos \left(\left(k_{1} x+l_{1} t\right)-i \phi_{2}\right)}{\sqrt{M} \cosh \left(l_{2} t+\phi\right)+\cos \left(k_{1} x+l_{1} t\right)} \\
q=q_{0} e^{-i(a t+\theta)} \frac{\sqrt{M} \cosh \left(l_{2} t+\phi+i \theta\right)+\cos \left(\left(k_{1} x+l_{1} t\right)-i \phi_{2}\right)}{\sqrt{M} \cosh \left(l_{2} t+\phi\right)+\cos \left(k_{1} x+l_{1} t\right)} \\
b=b_{0}-\frac{2\left(d_{1} \cosh \left(l_{2} t+\phi\right) \cos \left(k_{1} x+l_{1} t\right)+d_{2} \sinh \left(l_{2} t+\phi\right) \sin \left(k_{1} x+l_{1} t\right)+d_{3}\right)}{\left(\sqrt{M} \cosh \left(l_{2} t+\phi\right)+\cos \left(k_{1} x+l_{1} t\right)\right)^{2}}
\end{array}\right.
$$

where $\phi=\phi_{1}+r$. This solution tends to the plane-wave solution as $t \rightarrow \infty$. In this case, we find $M=1+\frac{4 k_{1}^{4}}{l_{2}^{2}}$. Therefore we see that (2.7) has no poles and should be well behaved everywhere. So the solution (2.7) is a nonsingular solution. We have plotted the solution (2.7) in Figure 3. This solution is periodic with period $\frac{2 \pi}{k_{1}}$ in $x$ and exponentially decaying, localized in $t$ direction. This spatially periodic breather solution is nothing but the Akhmediev breather solution. 


\subsubsection{Rogue wave}

To obtain a rogue wave from an inclined periodic breather, we consider the limit of the inclined periodic breather solution with the choice $l_{1}=k_{1} \lambda, l_{2}=-k_{2} \lambda, k_{1}^{2}=3 k_{2}^{2}, e^{r}=-1$. The propagation speed of the inclined periodic breather is given by $\lambda$. Substituting the above expressions into the inclined periodic breather form, (2.4) and rogue waves of the coupled long-wave-short-wave system are derived when the periods of the periodic rogue wave go to infinite. Indeed, by letting $k_{1} \rightarrow 0$ in (2.4), (2.4) becomes a rational solution

$$
\left\{\begin{array}{l}
p=p_{0} \mathrm{e}^{-i a t}\left(1-\frac{\frac{16 i}{\lambda}(x-\lambda t)+64 \lambda^{-2}}{\left(4\left(x+\frac{\lambda}{2} t\right)^{2}+3 t^{2} \lambda^{2}+\frac{64}{3 \lambda^{2}}\right)}\right), \\
q=q_{0} \mathrm{e}^{-i a t}\left(1-\frac{\frac{16 i}{\lambda}(x-\lambda t)+64 \lambda^{-2}}{\left(4\left(x+\frac{\lambda}{2} t\right)^{2}+3 t^{2} \lambda^{2}+\frac{64}{3 \lambda^{2}}\right)}\right) \\
b=b_{0}-\frac{16}{\left(4\left(x+\frac{\lambda}{2} t\right)^{2}+3 \lambda^{2} t^{2}+\frac{64}{3 \lambda^{2}}\right)}+\frac{128\left(x+\frac{\lambda}{2} t\right)^{2}}{\left(4\left(x+\frac{\lambda}{2} t\right)^{2}+3 \lambda^{2} t^{2}+\frac{64}{3 \lambda^{2}}\right)^{2}}
\end{array}\right.
$$

under the following condition:

$$
64 p_{0} q_{0}+\lambda^{3}=0
$$

This solution is nothing but the rogue wave solution of a coupled long-wave-short-wave system which is localized both in space and time. The typical spatial-temporal structure of the rogue wave is shown in Figure 4. From (2.8), the denominators of this family of solution are clearly nonsingular. This solution is well behaved everywhere. The maximum amplitude of the rogue wave solution $|p|^{2}$ occurs at the point $(0,0)$ and the maximum amplitude of this rogue wave solution is equal to $4 p_{0}^{2}$. The minimum amplitude of $|p|^{2}$ occurs at two points $\left(t= \pm \frac{4 \sqrt{2}}{3 \lambda^{2}}, x= \pm \frac{4 \sqrt{2}}{3 \lambda}\right)$, and the minimum amplitude of this rogue wave solution is equal to 0 . A similar result occurs for $|q|^{2}$ (not shown here). The maximum amplitude of the rogue wave solution $b$ occurs at two points $\left(t=0, x= \pm \frac{4}{\lambda}\right)$ and the maximum amplitude of this rogue wave solution is equal to $b_{0}+\frac{3}{32} \lambda^{2}$. The minimum amplitude of $b$ occurs at the point $(t=0, x=0)$, and the minimum amplitude of this rogue wave solution is equal to $b_{0}-\frac{3}{4} \lambda^{2}$. It is easy to verify that $(p, q, b)$ is a solution of $(1.1)$. Moreover, $(p, q, b)$ is also a rational homoclinic solution and tends to the fixed cycle $\left(p_{0} e^{-i a t}, q_{0} e^{-i a t}, b_{0}\right)$ as $t \rightarrow \infty$ or $x \rightarrow \infty$. In fact, $(p, q, b) \rightarrow\left(p_{0} e^{-i a t+i \pi}, q_{0} e^{-i a t+i \pi}, b_{0}\right)$ when $t$ or $x \rightarrow-\infty$, and the cycles $\left(p_{0} e^{-i a t+i \pi}, q_{0} e^{-i a t+i \pi}, b_{0}\right)$ and $\left(p_{0} e^{-i a t}, q_{0} e^{-i a t}, b_{0}\right)$ were one and the same. This shows that $(p, q, b)$ is also a homoclinic rogue wave solution of a coupled long-wave-short-wave system, $p, q$ are bright homoclinic rogue waves and $b$ is a dark (see Figure 4). Moreover, it follows from Figure 4 that, as time goes to $\pm \infty$, this rogue wave develops a localized hump with a peak amplitude of more than three times the non-zero constant background in the intermediate times. It is shown that the rogue wave arises from the non-zero constant background and then disappears into the non-zero constant background again. By comparing with known results $[10,11,27]$, one finds that they are similar in structure. These solutions distinguish themselves in zero-amplitude points and in a tilted angle (see Figure 4).

Obviously, the center of solution (2.8) is located at a fixed point $(0,0)$ on the $(x, t)$ plane, that is, this rogue wave given by (2.8) reaches its minimum or maximum at fixed center $(0,0)$. Indeed, we can obtain a rogue wave with a controllable center under the transformation of coordinate translation $t \rightarrow t+\alpha, x \rightarrow x+\beta$. In this case, the rogue wave given 


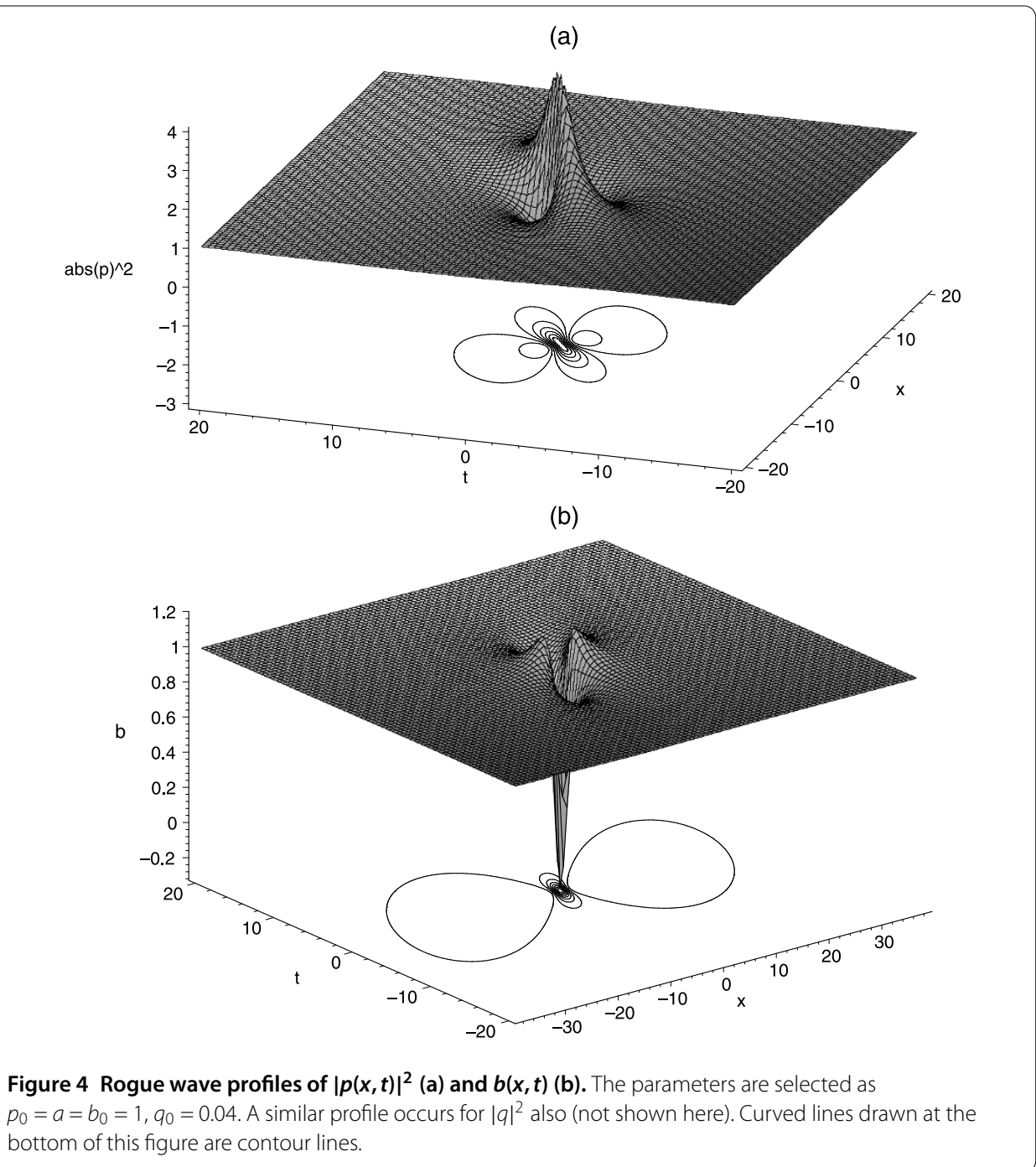

by (2.8) reaches its minimum or maximum at point $(\alpha, \beta)$, which is a controllable center on the $(x, t)$ plane.

\section{Conclusion and discussion}

In this paper, the Hirota two-soliton method has been applied to the coupled long-waveshort-wave system. Maple was used to compute the inclined periodic breather, Akhmediev breather, Ma breather and rogue wave solutions. Based on the structures of these rogue wave solutions and figures via computer simulation, characteristics of rogue wave solutions are discussed which might provide us with the useful information on the dynamics of the relevant physical fields. Following these ideas in this work, further study may be needed to see whether (1.1) has another type of specially spatiotemporal structure of the solutions. Moreover, we remark that these exact breather solutions belong to the class of solutions which the multiple exp-function algorithm [25] produces. The multiple expfunction algorithm is a generalization of Hirota's method. Recently, the Hirota bilinear method has also been generalized to more general bilinear equations by Ma [29]. 


\section{Competing interests}

The authors declare that they have no competing interests.

\section{Authors' contributions}

All authors contributed equally to the manuscript and read and approved the final manuscript.

\section{Author details}

${ }^{1}$ Department of Mathematics, Kunming University of Science and Technology, Kunming, Yunnan 650500, P.R. China.

${ }^{2}$ School of Mathematics and Physics, Yunnan University, Kunming, Yunnan 650500, P.R. China.

\section{Acknowledgements}

This work was supported by Chinese Natural Science Foundation Grant Nos. 11361048, 11301235, and 11261049, Yunnan Natural Science Foundation Grant No. kksy201307141.

\section{Received: 18 November 2013 Accepted: 26 February 2014 Published: 14 Mar 2014}

\section{References}

1. Ablowitz, MJ, Clarkson, PA: Solitons, Nonlinear Evolution Equations and Inverse Scattering. Cambridge University Press, Cambridge (1991)

2. Kharif, C, Pelinovsky, E, Slunyaev, A: Rogue Waves in the Ocean, Observation, Theories and Modeling. Springer, New York (2009)

3. Ganshin, AN, Efimov, VB, Kolmakov, GV, Mezhov-Deglin, LP, McClintock, PVE: Observation of an inverse energy cascade in developed acoustic turbulence in superfluid helium. Phys. Rev. Lett. 101, 065303 (2008)

4. Bludov, VYu, Konotop, WV, Akhmediev, N: Matter rogue waves. Phys. Rev. A 80, 033610 (2009)

5. Montina, A, Bortolozzo, U, Residori, S, Arecchi, FT: Non-Gaussian statistics and extreme waves in a nonlinear optical cavity. Phys. Rev. Lett. 103, 173901 (2009)

6. Bailung, H, Sharma, SK, Nakamura, Y: Observation of Peregrine solitons in a multicomponent plasma with negative ions. Phys. Rev. Lett. 107, 255005 (2011)

7. Yan, ZY: Financial rogue waves. Commun. Theor. Phys. 5, 947 (2010)

8. Akhmediev, N, Ankiewicz, A: Solitons, Nonlinear Pulses and Beams. Chapman \& Hall, London (1997)

9. Agrawal, GP: Nonlinear Fiber Optics. Academic Press, New York (2001)

10. Peregrine, DH: Water waves, nonlinear Schrödinger equations and their solutions. J. Aust. Math. Soc. Ser. B, Appl. Math 25, 16 (1983)

11. Akhmediev, N, Ankiewicz, A, Soto-Crespo, JM: Rogue waves and rational solutions of the nonlinear Schrödinger equation. Phys. Rev. E 80, 026601 (2009)

12. $\mathrm{Xu}, \mathrm{S}, \mathrm{He}$, J: The Darboux transformation of the derivative nonlinear Schrödinger equation. J. Phys. A, Math. Theor. 53, 063507 (2013)

13. Tao, Y, He, J: Multisolitons, breathers, and rogue waves for the Hirota equation generated by the Darboux transformation. Phys. Rev. E 85, 026601 (2012)

14. Bandelow, U, Akhmediev, N: Persistence of rogue waves in extended nonlinear Schrödinger equations: integrable Sasa-Satsuma case. Phys. Lett. A 376, 1558 (2012)

15. Ohta, Y, Yang, J: Dynamics of rogue waves in the Davey-Stewartson II equation. J. Phys. A, Math. Theor. 46, 105202 (2013)

16. Zhao, LC, Liu, J: Rogue-wave solutions of a three-component coupled nonlinear Schrödinger equation. Phys. Rev. E 87, $013201(2013)$

17. Zhong, WP: Wave solutions of the generalized one-dimensional Gross-Pitaevskii equation. J. Nonlinear Opt. Phys. Mater. 21, 1250026 (2012)

18. Li, C, He, J, Porseizan, K: Rogue waves of the Hirota and the Maxwell-Bloch equation. Phys. Rev. E 87, 012913 (2013)

19. Djordjevic, VD, Redekopp, LG: On two-dimensional packets of capillary-gravity waves. J. Fluid Mech. 79, 703 (1977)

20. Benney, DJ: A general theory for interactions between short and long waves. Stud. Appl. Math. 56, 81 (1977)

21. Grimshaw, RHJ: The modulation of an internal gravity-wave packet, and the resonance with the mean motion. Stud. Appl. Math. 56, 241 (1977)

22. Wright, OC: On a homoclinic manifold of a coupled long-wave-short-wave system. Commun. Nonlinear Sci. Numer. Simul. 15, $2066(2010)$

23. Tajiri, M, Takeuchi, K, Arai, T: Asynchronous development of the Benjamin-Feir unstable mode. Phys. Rev. E 64, 056622 (2001)

24. Tajiri, M, Arai, T: Quasi-line soliton interactions of the Davey-Stewartson I equation: on the existence of long-range interaction between two quasi-line solitons through a periodic soliton. J. Phys. A, Math. Theor. 44, 235204 (2011)

25. Ma, WX, Zhu, ZN: Solving the (3 + 1)-dimensional generalized KP and BKP equations by the multiple exp-function algorithm. Appl. Math. Comput. 218, 11871 (2012)

26. $\mathrm{Ma}, \mathrm{WX}$ : Complexiton solutions to integrable equations. Nonlinear Anal. 63, e2461 (2005)

27. Chabchoub, A, Hoffmann, N, Onorato, M, Akhmediev, N: Super rogue waves: observation of a higher-order breather in water waves. Phys. Rev. E 2, 011015 (2012)

28. Chabchoub, A, Hoffmann, NP, Akhmediev, N: Rogue wave observation in a water wave tank. Phys. Rev. E 106, 204502 (2011)

29. Ma, WX: Bilinear equations and resonant solutions characterized by Bell polynomials. Rep. Math. Phys. 72, 41 (2013)

10.1186/1687-1847-2014-87

Cite this article as: Wang and Dai: Various breathers and rogue waves for the coupled long-wave-short-wave system. Advances in Difference Equations 2014, 2014:87 